\title{
THE CAUCHY FUNCTIONAL EQUATIONS IN DISTRIBUTIONS
}

\author{
E. L. $\mathrm{KOH}$
}

(Communicated by Kenneth R. Meyer)

\begin{abstract}
The Pompeiu functional equation is defined by Neagu for Schwartz distributions. His method is extended to the four Cauchy functional equations by means of two new operators $Q^{*}$ and $R^{*}$ on $\mathscr{D}^{\prime}(I)$. The Cauchy equations in distributions reduce to the classical equations when the solutions are regular distributions, i.e. locally integrable functions.
\end{abstract}

\section{INTRODUCTION}

Recently [1], the Pompeiu equation has been defined for distributions in $\mathscr{D}^{\prime}(\Omega)$ where $\Omega=(-1, \infty) \subset \mathbf{R}$. When the distribution is a locally integrable function $f(x)$, the equation in distribution reduces to the classical Pompeiu equation

$$
f(x+y+x y)=f(x)+f(y)+f(x) f(y) .
$$

Fenyö [2], in finding the most general solution to the Hosszú equation

$$
f(x+y-x y)+f(x y)=f(x)+f(y),
$$

has defined and solved it in distributional form. In this paper, we define the Cauchy equations in distributions by means of two new operators $Q^{*}$ and $R^{*}$ on $\mathscr{D}^{\prime}(I)$. When the solutions are locally integrable functions, the equations reduce to the classical Cauchy functional equations [3].

$$
\begin{aligned}
f(x+y) & =f(x)+f(y) \\
f(x+y) & =f(x) f(y) \\
f(x y) & =f(x)+f(y) \\
f(x y) & =f(x) f(y) .
\end{aligned}
$$

\section{Preliminaries}

Let $I=(0, \infty) \subset \mathbf{R}$ and $I^{2}=I \times I \subset \mathbf{R}^{2}$. We denote by $\mathscr{D}(I)$ and $\mathscr{D}\left(I^{2}\right)$ the spaces of infinitely differentiable functions on $I$ and $I^{2}$ with compact supports, respectively. Similarly, $\mathscr{E}(I)$ and $\mathscr{E}\left(I^{2}\right)$ are spaces of infinitely

Received by the editors March 6, 1988.

1980 Mathematics Subject Classification (1985 Revision). Primary 46F10, 39B70. 
differentiable functions on $I$ and $I^{2}$. The duals of these spaces are denoted by a prime, e.g. $\mathscr{D}^{\prime}(I)$. Note that $\mathscr{D}(I) \subset \mathscr{E}(I) \subset \mathscr{E}^{\prime}(I) \subset \mathscr{D}^{\prime}(I)$ (see [4]). We denote by $L_{\text {loc }}(I)$ and $L_{\text {loc }}\left(I^{2}\right)$ the spaces of locally integrable functions on $I$ and $I^{2}$, respectively.

$D$ is the differentiation operator in $\mathscr{D}^{\prime}(I)$, whereas $D_{1}$ and $D_{2}$ are the partial differentiation operators in $\mathscr{D}^{\prime}\left(I^{2}\right)$ with respect to the first and second variable from $I^{2}$, respectively.

\section{SOME OPERATORS ON $\mathscr{D}^{\prime}$}

Let $E_{1}$ and $E_{2}$ be integration operators from $\mathscr{D}\left(I^{2}\right)$ into $\mathscr{D}(I)$ given, respectively, by

$$
E_{1}[\phi](x)=\int_{I} \phi(x, y) d y
$$

and

$$
E_{2}[\phi](y)=\int_{I} \phi(x, y) d x
$$

for any $\phi \in \mathscr{D}\left(I^{2}\right)$. It is easy to see that both of (5) and (6) are linear operators and we denote this by membership in $\mathscr{L}\left[\mathscr{D}\left(I^{2}\right) ; \mathscr{D}(I)\right]$. The adjoints of $E_{1}$ and $E_{2}$ are the operators $E_{1}^{*}$ and $E_{2}^{*}$ from $\mathscr{D}^{\prime}(I)$ into $\mathscr{D}^{\prime}\left(I^{2}\right)$ defined by

$$
\begin{aligned}
& \left\langle E_{1}^{*}[T], \phi\right\rangle=\left\langle T, E_{1} \phi\right\rangle=\left\langle T_{x}, \int_{I} \phi(x, y) d y\right\rangle \\
& \left\langle E_{2}^{*}[T], \phi\right\rangle=\left\langle T, E_{2} \phi\right\rangle=\left\langle T_{y}, \int_{I} \phi(x, y) d x\right\rangle
\end{aligned}
$$

for $T \in \mathscr{D}^{\prime}(I)$ and $\phi \in \mathscr{D}\left(I^{2}\right)$. We note that $E_{i}^{*} \in \mathscr{L}\left[\mathscr{D}^{\prime}(I) ; \mathscr{D}^{\prime}\left(I^{2}\right)\right]$, $i=1,2$. The following is proved in an analogous way to [1] after replacing the domains $\Omega$ and $\Omega^{2}$ by $I$ and $I^{2}$, respectively,

Proposition 1.

(a) If $f \in L_{\mathrm{loc}}(I)$, then $g=E_{1}^{*}[f] \in L_{\mathrm{loc}}\left(I^{2}\right)$ and $h=E_{2}^{*}[f] \in L_{\mathrm{loc}}\left(I^{2}\right)$, where $g(x, y)=f(x)$ and $h(x, y)=f(y)$.

(b) If $\alpha \in \mathscr{E}(I)$, then $\beta=E_{1}^{*}[\alpha] \in \mathscr{E}\left(I^{2}\right)$ and $\gamma=E_{2}^{*}[\alpha] \in \mathscr{E}\left(I^{2}\right)$.

(c) If $\alpha \in \mathscr{E}(I)$ and $T \in \mathscr{D}^{\prime}(I)$, then

$$
E_{i}^{*}[\alpha T]=E_{i}^{*}[\alpha] E_{i}^{*}[T], \quad i=1,2 .
$$

(d) If $T \in \mathscr{D}^{\prime}(I)$, then

$$
\begin{aligned}
& D_{1} E_{1}^{*}[T]=E_{1}^{*}[D T] ; \quad D_{1} E_{2}^{*}[T]=0 \\
& D_{2} E_{1}^{*}[T]=0 ; \quad D_{2} E_{2}^{*}[T]=E_{2}^{*}[D T] .
\end{aligned}
$$

Let $P$ be the direct product operator from $\mathscr{D}^{\prime}(I) \times \mathscr{D}^{\prime}(I)$ into $\mathscr{D}^{\prime}\left(I^{2}\right)$ given by

$$
\langle P[S ; T], \phi\rangle=\left\langle S_{x},\left\langle T_{y}, \phi(x, y)\right\rangle\right\rangle=\left\langle T_{y},\left\langle S_{x}, \phi(x, y)\right\rangle\right\rangle
$$


for any $S, T \in \mathscr{D}^{\prime}(I)$ and $\phi \in \mathscr{D}\left(I^{2}\right)$. It is known that $P$ is a linear operator and that

$$
D_{1} P[S ; T]=P[D S ; T] ; \quad D_{2} P[S ; T]=P[S ; D T]
$$

(see [4]). The following result from [1] is immediate, mutatis mutandis. (Note that equations (14) as stated in [1] are incorrect.)

\section{Proposition 2.}

(a) If $T \in \mathscr{D}^{\prime}(I)$, then we have

$$
E_{1}^{*}[T]=P[T ; 1] ; \quad E_{2}^{*}[T]=P[1 ; T] .
$$

(b) If $\alpha, \beta \in \mathscr{E}(I)$ and $S, T, U \in \mathscr{D}^{\prime}(I)$, then we have

$$
\begin{aligned}
& E_{1}^{*}[\alpha] P[S ; U]+E_{1}^{*}[\beta] P[T ; U]=P[\alpha S+\beta T ; U] \\
& E_{2}^{*}[\alpha] P[S ; T]+E_{2}^{*}[\beta] P[S ; U]=P[S ; \alpha T+\beta U] .
\end{aligned}
$$

(c) If $S, T, U, V \in \mathscr{D}^{\prime}(I)$ are nonzero distributions and satisfy the equation

$$
P[S ; T]=P[U ; V],
$$

then $S=c_{1} U$ and $T=c_{2} V$ (where $c_{1}$ and $c_{2}$ are real constants) and conversely, (17) is satisfied for all real $c_{1}$ and $c_{2}$ such that $c_{1}=1 / c_{2}$.

\section{THE OPERATORS $Q^{*}$ AND $R^{*}$}

Let $Q$ and $R$ be operators from $\mathscr{D}\left(I^{2}\right)$ into $\mathscr{D}(I)$ given by

$$
\begin{gathered}
Q[\phi](u)=\int_{I} \phi(u-v, v) d v=\int_{I} \phi(v, u-v) d v \\
R[\phi](u)=\int_{I} \frac{\phi(u / v, v)}{v} d v=\int_{I} \frac{\phi(v, u / v)}{v} d v .
\end{gathered}
$$

These are well-defined since $\phi(x, y)$ has compact support. We note that $Q, R$ $\in \mathscr{L}\left[\mathscr{D}\left(I^{2}\right) ; \mathscr{D}(I)\right]$. The adjoints of these operators are $Q^{*}, R^{*} \in \mathscr{L}\left[\mathscr{D}^{\prime}(I)\right.$; $\left.\mathscr{D}^{\prime}\left(I^{2}\right)\right]$ defined by

$$
\begin{aligned}
& \left\langle Q^{*}[T], \phi\right\rangle=\langle T, Q[\phi]\rangle=\left\langle T_{u}, Q[\phi](u)\right\rangle \\
& \left\langle R^{*}[T], \phi\right\rangle=\langle T, R[\phi]\rangle=\left\langle T_{u}, R[\phi](u)\right\rangle .
\end{aligned}
$$

\section{Proposition 3.}

(a) If $f \in L_{\mathrm{loc}}(I)$, then $Q^{*}[f] \in L_{\mathrm{loc}}\left(I^{2}\right)$ where $Q^{*} f=f(x+y)$.

(b) If $f \in L_{\mathrm{loc}}(I)$, then $R^{*}[f] \in L_{\mathrm{loc}}\left(I^{2}\right)$ where $R^{*} f=f(x y)$.

Proof. (a) For any $\phi \in \mathscr{D}\left(I^{2}\right)$, we have

$$
\begin{aligned}
\left\langle Q^{*}[f], \phi\right\rangle & =\langle f, Q[\phi]\rangle=\left\langle f(u), \int_{I} \phi(u-v, v) d v\right\rangle \\
& =\int_{I^{2}} f(u) \phi(u-v, v) d v d u \\
& =\langle f(x+y), \phi(x, y)\rangle .
\end{aligned}
$$


(b) For any $\phi \in \mathscr{D}\left(I^{2}\right)$, we have

$$
\begin{aligned}
\left\langle R^{*}[f], \phi\right\rangle & =\left\langle f(u), \int_{I} \frac{\phi(u / v, v)}{v} d v\right\rangle \\
& =\int_{I^{2}} \frac{f(u) \phi(u / v, v)}{v} d v d u \\
& =\langle f(x y), \phi(x, y)\rangle .
\end{aligned}
$$

Proposition 4. If $\alpha(x) \in \mathscr{E}(I)$, then both $Q^{*}(\alpha)$ and $R^{*}(\alpha)$ belong to $\mathscr{E}\left(I^{2}\right)$.

Proof. Analogous to Proposition 3.

Proposition 5. If $\alpha \in \mathscr{E}(I)$ and $T \in \mathscr{D}^{\prime}(I)$, then

$$
\begin{aligned}
& Q^{*}[\alpha T]=Q^{*}[\alpha] Q^{*}[T] \\
& R^{*}[\alpha T]=R^{*}[\alpha] R^{*}[T]
\end{aligned}
$$

Proof. For any $\phi \in \mathscr{D}\left(I^{2}\right)$, we have

$$
\begin{aligned}
\left\langle R^{*}[\alpha T], \phi\right\rangle & =\langle\alpha T, R[\phi]\rangle=\langle T, \alpha R[\phi]\rangle \\
& =\left\langle T, R\left[\phi R^{*}[\alpha]\right]\right\rangle=\left\langle R^{*}[T], \phi R^{*}[\dot{\alpha}]\right\rangle \\
& =\left\langle R^{*}[\alpha] R^{*}[T], \phi\right\rangle
\end{aligned}
$$

The first statement is proved in the same way.

Proposition 6. If $T \in \mathscr{D}^{\prime}(I)$, then

$$
\begin{gathered}
D_{1}\left(Q^{*}[T]\right)=Q^{*}(D T)=D_{2}\left(Q^{*}[T]\right) \\
D_{1}\left(R^{*}[T]\right)=E_{2}^{*}(\Omega) R^{*}(D T) \\
D_{2}\left(R^{*}[T]\right)=E_{1}^{*}(\Omega) R^{*}(D T)
\end{gathered}
$$

where $\Omega=x \in \mathscr{E}(I)$.

Proof. Let $\phi(x, y) \in \mathscr{D}\left(I^{2}\right)$ and $\psi(x, y)=-\partial \phi / \partial x$. Then

$$
\begin{aligned}
\left\langle D_{1}\left(Q^{*}[T]\right), \phi(x, y)\right\rangle & =\left\langle Q^{*}[T], \psi(x, y)\right\rangle=\langle T, Q \psi\rangle \\
& =\left\langle T_{u}, \int_{I} \psi(u-v, v) d v\right\rangle=\left\langle T_{u}, \int_{I}-\frac{\partial \phi}{\partial u}(u-v, v) d v\right\rangle \\
& =\left\langle T_{u},-\frac{\partial}{\partial u} \int_{I} \phi(u-v, v) d v\right\rangle \\
& =\langle D T, Q(\phi)\rangle=\left\langle Q^{*}[D T], \phi\right\rangle .
\end{aligned}
$$


Since $\int_{I} \phi(u-v, v) d v=\int_{I} \phi(v, u-v) d v$, the second part of (24) is proved analogously. To prove (25), we have

$$
\begin{aligned}
\left\langle D_{1}\left(R^{*}[T]\right), \phi(x, y)\right\rangle & =\left\langle R^{*}[T], \psi(x, y)\right\rangle=\langle T, R \psi\rangle \\
& =\left\langle T_{u}, \int_{I} \frac{\psi(u / v, v)}{v} d v\right\rangle \\
& =\left\langle T_{u}, \int_{I} \frac{-v}{v} \frac{\partial \phi}{\partial u}\left(\frac{u}{v}, v\right) d v\right\rangle \\
& =\left\langle T_{u}, \frac{-\partial}{\partial u} \int_{I} \phi\left(\frac{u}{v}, v\right) d v\right\rangle=\left\langle D T_{u}, R[\phi v]\right\rangle \\
& =\left\langle R^{*}[D T], \phi \cdot E_{2}^{*}(\Omega)\right\rangle \quad \text { where } \Omega=x \in \varepsilon(I) \\
& =\left\langle E_{2}^{*}(\Omega) R^{*}[D T], \phi\right\rangle .
\end{aligned}
$$

Equation (26) is proved analogously.

\section{The Cauchy equations in Distributions}

Using the operators $Q^{*}$ and $R^{*}$, we shall call the following equations the Cauchy equations in distribution $T \in D^{\prime}(I)$ :

$$
\begin{aligned}
& Q^{*}[T]=E_{1}^{*}[T]+E_{2}^{*}[T] \\
& Q^{*}[T]=P[T ; T] \\
& R^{*}[T]=E_{1}^{*}[T]+E_{2}^{*}[T] \\
& R^{*}[T]=P[T ; T] .
\end{aligned}
$$

Proposition 7. If $T=f \in L_{\mathrm{loc}}(I)$, equations (27) to (30) reduce to the Cauchy functional equations (1) to (4), respectively.

Proof. The statement follows from Proposition 1(a), Proposition 3, and the fact that for $\phi \in \mathscr{D}\left(I^{2}\right)$,

$$
\begin{aligned}
\langle P[f ; f], \phi\rangle & =\langle f(x),\langle f(y), \phi(x, y)\rangle\rangle \\
& =\int_{I^{2}} f(x) f(y) \phi(x, y) d x d y \\
& =\langle f(x) f(y), \phi(x, y)\rangle .
\end{aligned}
$$

Theorem 1. If $T \in \mathscr{D}^{\prime}(I)$ satisfies (27), then $T \in \mathscr{E}(I)$ where $T=c \Omega, c \in \mathbf{R}$. Proof. From Propositions 6, 1(d) and 2(a), we have

$$
\begin{aligned}
& Q^{*}[D T]=D_{1} Q^{*}[T]=D_{1} E_{1}^{*}[T]+D_{1} E_{2}^{*}[T]=P[D T ; 1] \\
& Q^{*}[D T]=D_{2} Q^{*}[T]=D_{2} E_{1}^{*}[T]+D^{2} E_{2}^{*}[T]=P[1 ; D T] .
\end{aligned}
$$

Hence $P[1 ; D T]=P[D T ; 1]$ and by Proposition $2(\mathrm{c}), D T=c$ or $T=c \Omega$, $c \in \mathbf{R}$.

Corollary 1. If $f \in L_{\mathrm{loc}}(I)$ satisfies (27), then $f(x) \in \mathscr{E}(I)$ where $f(x)=c x$, $c \in \mathbf{R}$.

This is the classical solution of the Cauchy functional equation (1). 
Theorem 2. If $T \in \mathscr{D}^{\prime}(I)$ satisfies (28), then $T \in \mathscr{E}(I)$ where $T=e^{c \Omega}, c \in \mathbf{R}$ or $T \equiv 0$.

Proof. From Propositions 6, 1(d) and equation (13), we have

$$
\begin{aligned}
& Q^{*}[D T]=D_{1} Q^{*}[T]=D_{1} P[T ; T]=P[D T ; T] \\
& Q^{*}[D T]=D_{2} Q^{*}[T]=D_{2} P[T ; T]=P[T ; D T] .
\end{aligned}
$$

Hence $P[D T ; T]=P[T, D T]$ and by Proposition $2(\mathrm{c}), D T=c T$ or $T=$ $c_{1} e^{c \Omega}, c_{1}, c \in \mathbf{R}$. On substituting back into (28), we obtain $c_{1}=1$ or 0 . Thus $T=e^{c \Omega}$ or $T \equiv 0$.

Corollary 2. If $f \in L_{\text {loc }}(I)$ satisfies (28), then $f(x) \in \mathscr{E}(I)$ where $f(x)=e^{c x}$ or $0, c \in \mathbf{R}$.

Theorem 3. If $T \in D^{\prime}(I)$ satisfies (29), then $T \in \mathscr{E}(I)$ where $T=c \ln \Omega$, $c \in \mathbf{R}$.

Proof. From Propositions 6, 1(d) and 2(a), we have

$$
\begin{aligned}
& E_{2}^{*}(\Omega) R^{*}(D T)=D_{1} R^{*}[T]=D_{1} E_{1}^{*}[T]+D_{1} E_{2}^{*}[T]=P[D T ; 1] \\
& E_{1}^{*}(\Omega) R^{*}(D T)=D_{2} R^{*}[T]=D_{2} E_{1}^{*}[T]+D_{2} E_{2}^{*}[T]=P[1 ; D T] .
\end{aligned}
$$

Hence $E_{1}^{*}(\Omega) P[D T ; 1]=E_{2}^{*}(\Omega) P[1 ; D T]$. From Proposition 2(b) and 2(c), we obtain $\Omega D T=c, c \in \mathbf{R}$, Solving the differential equation in $T$, we get $T=c \ln \Omega+a$ for some arbitrary constant $a$. Substituting the last expression into the equation, we see that $a=0$ and the theorem is proved.

Corollary 3. If $f \in L_{\text {loc }}(I)$ satisfies (29), then $f(x) \in \mathscr{E}(I)$ where $f(x)=c \ln x$, $c \in \mathbf{R}$.

This is the well-known solution of the Cauchy equation (3). In a similar manner, the following result can easily be shown.

Theorem 4. If $T \in \mathscr{D}^{\prime}(I)$ satisfies (30), then $T \in \mathscr{E}(I)$ where $T=\Omega^{c}, c \in \mathbf{R}$ or $T \equiv 0$.

Corollary 4. If $f \in L_{\text {loc }}(I)$ satisfies (30), then $f(x) \in \mathscr{E}(I)$ where $f(x)=x^{c}$, $c \in \mathbf{R}$ or $f(x) \equiv 0$.

\section{REFERENCES}

1. M. Neagu, About the Pompeiu equation in distributions, Inst. Politehn. "Traian Vuia" Timisoara. Lucrăr. Sem. Mat. Fiz. (1984) May, 62-66.

2. I. Fenyö, On the general solution of a functional equation in the domain of distributions, Aequationes Math. 3 (1969), 236-246.

3. J. Aczel, Lectures on functional equations and their applications, Academic Press, New York, 1966.

4. L. Schwartz, Théorie des Distributions, Hermann, Paris, 1966.

Department of Mathematics and Statistics, University of Regina, Regina, CANADA S4S 0A2 